\section{US authorities uphold suspension of SCID gene therapy}

Ere rench researchers recently disclosed that a second child treated with a retrovirus vector in a gene therapy clinical trial at Hôpital Necker-Enfants Malades (Paris, France) developed leukemia (Nat. Biotechnol. 21, 121, 2003). After reviewing these two cases during a special meeting in February, members of the National Institutes of Health Recombinant DNA Advisory Committee (NIHRAC; Bethesda, $\mathrm{MD}$ ) are recommending that similar trials involving patients with $\mathrm{X}$-linked, severe combined immunodeficiency syndrome (X-SCID) continue to be suspended. However, they also indicated that other gene transfer clinical trials involving retroviruses could resume-provided, of course, that they meet criteria for safety, informed consent, and scientific worthiness.

The status of gene transfer and gene therapy clinical trials has lately been in considerable flux while members of NIHRAC and a similar advisory panel of the Food and Drug Administration (FDA; Rockville, $\mathrm{MD})$, called the Biological Response Modifiers Advisory Committee (BRMAC) conferred several times to interpret these "adverse event" findings in the French clinical trial (Nat. Biotechnol. 20, 1068, 2002). Indeed, BRMAC members were set to meet again to address the implications of this second case of leukemia at the end of February, while NIHRAC is scheduled to convene early in March for a broader review of retroviral vector safety issues.

NIHRAC members and FDA officials both recently recommended putting many US gene transfer experiments on clinical hold. The "second event" in France led FDA officials quickly to "enlarge the scope of the retroviral vector hold... without regard to disease" affecting patients participating in about 30 of the 150 ongoing clinical gene transfer trials, according to Philip Noguchi, who directs the Office of Cellular, Tissue, and Gene Therapies in the FDA Center for Biologics Evaluation and Research. Of those on hold, the original three involving several forms of X-SCID will remain suspended, whereas many of the other 27 that involve life-threatening diseases are being considered for restarting as new safety measures are being implemented, he says.

The mid-January NIHRAC recommendations were more restrictive than those from the FDA, with committee members urging a halt to all gene transfer protocols

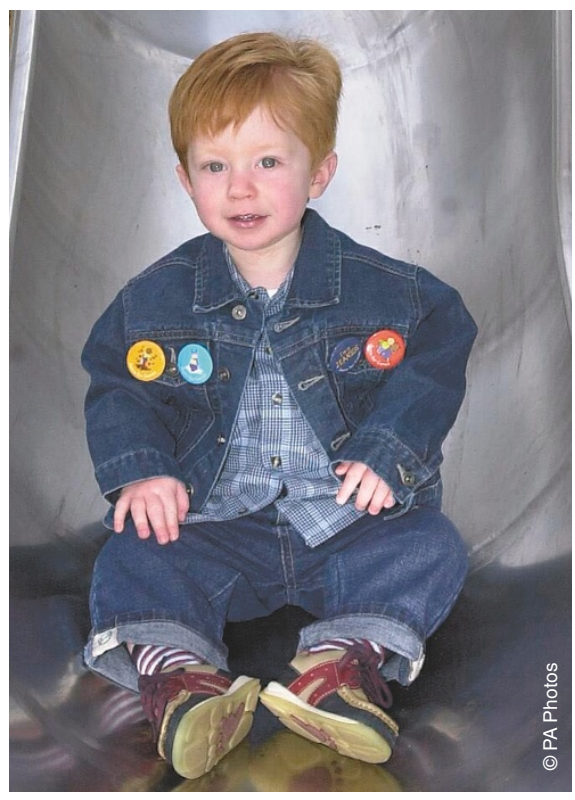

Rhys Evans became the first SCID patient to benefit from ground-breaking gene therapy study.

involving retroviruses targeting any kind of hematopoietic cell. Although FDA officials called for suspensions of clinical trials involving retroviral vectors, they focused more narrowly on suspending those that target progenitor, or stem, cells similar to those that were the target cells for the retroviral vectors used in the French XSCID trial.

\section{Many of the other 27 retroviral gene therapy trials that involve life-threatening diseases are being considered for restarting as new safety measures are being implemented.}

This seemingly slight difference between the NIH and FDA advisory messages proved consequential and frustrating for a number of investigators who were conducting a range of gene transfer trials, including several who called on NIHRAC to ease up on the restrictions and to make its follow-up advice very precise.
"IRBs [institutional review boards] are very skittish about gene therapy, don't have [comparable] expertise, and consider [NIHRAC] recommendations as law," says Steven Rosenberg of the National Cancer Institute at NIH. Thus, the mid-January advisory from NIHRAC effectively blocked his and several other similar protocols that entailed treating patients who have advanced cancer with their own genetically modified T cells. Such cancer patients appear to be at little risk for developing leukemia, in part, because their survival expectations are so bleak, Rosenberg points out. Thus, it took 30 months for leukemia to manifest clinically in each of the two children in France, and the mechanism underlying its development does not appear applicable to the molecular and cellular manipulations at work in the clinical protocols involving adults with cancer.

Noting that the two leukemia cases among the nine participants in the French clinical trail are "unprecedented" and "unanticipated," Ken Cornetta of Indiana University School of Medicine (Indianapolis, IN), who represents the American Society of Gene Therapy (Milwaukee, WI), called on the FDA and NIHRAC to "harmonize" their advice. "Harmony helps our investigators, who need to be compliant with recommendations from.... and must respond to six or more committees."

That initially restrictive advice from NIHRAC in mid-January to investigators was not offered "lightly," says committee member Diane Wara of the University of California, San Francisco. When committee members learned late in December of the second case of leukemia in France, they realized that an " $n=2$ ' heightened the risk... and eliminated the notion that it was...chance." Although that advice may have caused 'angst' among investigators conducting gene transfer protocols, it was intended as a stopgap or 'place-holder,' pending the deliberations of the full committee with outside experts during the special February meeting, adds Amy Patterson, who directs the NIH Office of Biotechnology Activities, through which NIHRAC operates.

Meanwhile, early in February an advisory commission in Germany similarly finetuned its advice regarding several gene therapy clinical protocols, according to Klaus Cichutek of the Paul-Ehrlich-Institut (Frankfurt, Germany). Although the commission imposed new safety restrictions, those restrictions were tailored to reflect the specific mechanism that apparently accounts for the two cases of leukemia in the French gene therapy trial.

Jeffrey L. Fox 